\title{
Modeling Naturalistic Affective States Via Facial, Vocal, and Bodily Expressions Recognition
}

\author{
Kostas Karpouzis ${ }^{1}$, George Caridakis ${ }^{1}$, Loic Kessous ${ }^{2}$, Noam Amir ${ }^{2}$, \\ Amaryllis Raouzaiou ${ }^{1}$, Lori Malatesta ${ }^{1}$, and Stefanos Kollias ${ }^{1}$ \\ ${ }^{1}$ Image, Video and Multimedia Systems Laboratory, National Technical University of Athens, \\ Politechnioupoli, Zographou, Greece \\ \{kkarpou, gcari, araouz, lori, stefanos\}@image.ntua.gr \\ ${ }^{2}$ Tel Aviv Academic College of Engineering \\ 218 Bnei Efraim St. 69107, Tel Aviv, Israel \\ \{kessous, noama\} @post.tau.ac.il
}

\begin{abstract}
Affective and human-centered computing have attracted a lot of attention during the past years, mainly due to the abundance of devices and environments able to exploit multimodal input from the part of the users and adapt their functionality to their preferences or individual habits. In the quest to receive feedback from the users in an unobtrusive manner, the combination of facial and hand gestures with prosody information allows us to infer the users' emotional state, relying on the best performing modality in cases where one modality suffers from noise or bad sensing conditions. In this paper, we describe a multi-cue, dynamic approach to detect emotion in naturalistic video sequences. Contrary to strictly controlled recording conditions of audiovisual material, the proposed approach focuses on sequences taken from nearly real world situations. Recognition is performed via a 'Simple Recurrent Network' which lends itself well to modeling dynamic events in both user's facial expressions and speech. Moreover this approach differs from existing work in that it models user expressivity using a dimensional representation of activation and valence, instead of detecting discrete 'universal emotions', which are scarce in everyday human-machine interaction. The algorithm is deployed on an audiovisual database which was recorded simulating human-human discourse and, therefore, contains less extreme expressivity and subtle variations of a number of emotion labels.
\end{abstract}

Keywords: Affective interaction, multimodal analysis, facial expressions, prosody, hand gestures, neural networks.

\section{Introduction}

The introduction of the term 'affective computing' by R. Picard [45] epitomizes the fact that computing is no longer considered a 'number crunching' discipline, but should be thought of as an interfacing means between humans and machines and sometimes even between humans alone. To achieve this, application design must take into account the ability of humans to provide multimodal input to computers, thus moving away from the monolithic window-mouse-pointer interface paradigm and 
utilizing more intuitive concepts, closer to human niches ([1], [4]). A large part of this naturalistic interaction concept is expressivity [46], both in terms of interpreting the reaction of the user to a particular event or taking into account their emotional state and adapting presentation to it, since it alleviates the learning curve for conventional interfaces and makes less technology-savvy users feel more comfortable. In this framework, both speech and facial expressions are of great importance, since they usually provide a comprehensible view of users' reactions.

The complexity of the problem relies in the combination of the information extracted from modalities, the interpretation of the data through time and the noise alleviation from the natural setting. The current work aims to interpret sequences of events thus modeling the user's behavior through time. With the use of a recurrent neural network, the short term memory, provided through its feedback connection, works as a memory buffer and the information remembered is taken under consideration in every next time cycle. Theory on this kind of network back up the claim that it is suitable for learning to recognize and generate temporal patterns as well as spatial ones [20].

The naturalistic data chosen as input is closer to human reality since the dialogues are not acted, and the expressivity is not guided by directives (e.g. Neutral expression $\rightarrow$ one of the six universal emotions $\rightarrow$ neutral). This amplifies the difficulty in discerning facial expressions and speech patterns. Nevertheless it provides the perfect test-bed for the combination of the conclusions drawn from each modality in one time unit and use as input in the following sequence of audio and visual events analyzed.

In the area of unimodal emotion recognition there have been many studies using different, but single, modalities. Facial expressions [21], [50], [31], vocal features [41], [24] and physiological signals [48] have been used as inputs during these attempts, while multimodal emotion recognition is currently gaining ground $(26,28$, 32, 53).

A wide variety of machine learning techniques have been used in emotion recognition approaches ([50], [31], [42]). Especially in the multimodal case [34], they all employ a large number of audio, visual or physiological features, a fact which usually impedes the training process; therefore, we need to find a way to reduce the number of utilized features by picking out only those related to emotion. An obvious choice for this is neural networks, since they enable us to pinpoint the most relevant features with respect to the output, usually by observing their weights. Although such architectures have been successfully used to solve problems that require the computation of a static function, where output depends only upon the current input, and not on any previous inputs, this is not the case in the domain of emotion recognition. One of the reasons for this is that expressivity is a dynamic, time-varying concept, where it is not always possible to deduce an emotional state merely by looking at a still image. As a result, Bayesian approaches which lend themselves nicely to similar problems [37], need to be extended to include support for timevarying features. Picard [46] and Cohen [16] propose the use of Hidden Markov Models (HMMs) to model discrete emotional states (interest, joy or distress) and use them to predict the probability of each one, given a video of a user. However, this process needs to build a single HMM for each of the examined cases (e.g. each of the universal emotions), making it more suitable in cases where discrete emotions need to be estimated. In our case building dedicated HMMs for each of the quadrants of the 
emotion representation would not perform well, since each of them contains emotions expressed with highly varying features (e.g. anger and fear both lie in the negative/active quadrant) which cannot be modeled using a single model.

A more suitable choice would be RNNs (Recurrent Neural Networks) where past inputs influence the processing of future inputs [35]. RNNs possess the nice feature of modeling explicitly time and memory ([49], [14], [9]), catering for the fact that emotional states are not fluctuating strongly, given a short period of time. Additionally, they can model emotional transitions and not only static emotional representations, providing a solution for diverse feature variation and not merely for neutral to expressive and back to neutral, as would be the case for HMMs.

\section{Induction of Natural Emotions - Data Collection}

Research on signs of emotion emerged as a technical field around 1975, with research by Ekman and his colleagues [38] on encoding emotion-related features of facial expression, and by Williams and Stevens [52] on emotion in the voice. The early paradigms simplified their task by concentrating on emotional extremes - often simulated, and not always by skilled actors. Most of the data used in research on speech and emotion has three characteristics: the emotion in it is simulated by an actor (not necessarily trained); the actor is reading preset material; and he or she is aiming to simulate full-blown emotion.

That kind of material has obvious attractions: it is easy to obtain, and it lends itself to controlled studies. However, it has become reasonably clear that it does not do a great deal to illuminate the way face and speech express emotion in natural settings. The 1990's saw growing interest in naturalistic data, but retained a focus on cases where emotion was at or approaching an extreme. The major alternative is to develop techniques which might be called directed elicitation - techniques designed to induce states that are both genuinely emotional and likely to involve speech.

Most of these tasks have a restricted range. They provide more control and higher data rates than other methods, but they still tend to elicit weak negative emotions, and they often impose constraints on the linguistic form and content of the speech which may restrict generalizationlla. One of them, SAL, was used to acquire the data processed by the presented system.

The SAL scenario [42] is a development of the ELIZA concept introduced by Weizenbaum [19]. The user communicates with a system whose responses give the impression of sympathetic understanding, and that allows a sustained interaction to build up. In fact, though, the system takes no account of the user's meaning: it simply picks from a repertoire of stock responses on the basis of surface cues extracted from the user's contributions. A second factor in the selection is that the user selects one of four 'artificial listeners' to interact with at any given time. Each one will try to initiate discussion by providing cues mapped to each of the four quadrants defined by valence and activation - 'Spike' is provocative or angry (negative/active), while 'Poppy' is always happy (positive/active). SAL took its present form as a result of a good deal of pilot work [10]. In that form, it provides a framework within which users do express a considerable range of emotions in ways that are virtually unconstrained. The process depends on users' co-operation - they are told that it is like an emotional gym, and 
they have to use the machinery it provides to exercise their emotions. But if they do enter into the spirit, they can move through a very considerable emotional range in a recording session or a series of recording sessions: the 'artificial listeners' are designed to let them do exactly that.

As far as emotion representation is concerned we use the Activation-Evaluation space. Activation-Evaluation space as a representation has great appeal as it is both simple, while at the same time makes it possible to capture a wide range of significant issues in emotion. The concept is based on a simplified treatment of two key themes:

- Valence: The clearest common element of emotional states is that the person is materially influenced by feelings that are valenced, i.e., they are centrally concerned with positive or negative evaluations of people or things or events.

- Activation level: Research from Darwin forward has recognized that emotional states involve dispositions to act in certain ways. A basic way of reflecting that theme turns out to be surprisingly useful. States are simply rated in terms of the associated activation level, i.e., the strength of the person's disposition to take some action rather than none.

Dimensional representations are attractive mainly because they provide a way of describing emotional states that is more tractable than using words. This is of particular importance when dealing with naturalistic data, where a wide range of emotional states occur. Similarly, they are much more able to deal with non-discrete emotions and variations in emotional state over time, since it such cases changing from one universal emotion label to another would not make much sense in real life scenarios.

The available SAL Data Set is given along with some of their important features in Table 1, while Figure 1 shows some frames of the processed naturalistic data.

Table 1. Summary of the processed SAL data

\begin{tabular}{|c|c|}
\hline \multicolumn{2}{|c|}{ Data Set } \\
\hline Subjects & 2 males, 2 females \\
\hline Passages & 76 \\
\hline Tunes & $\sim 1600$ \\
\hline Emotion space coverage & Yes, all quadrants \\
\hline FeelTrace ratings & 4 rators \\
\hline Transcripts & Yes \\
\hline Text Post-Processing & No \\
\hline
\end{tabular}

Naturalistic data goes beyond extreme emotions, as is usually the case in existing approaches (see Fig. 1 for examples of expressive images, not so easy to classify using universal emotions), and concentrates on more natural emotional episodes that happen more frequently in everyday discourse. 

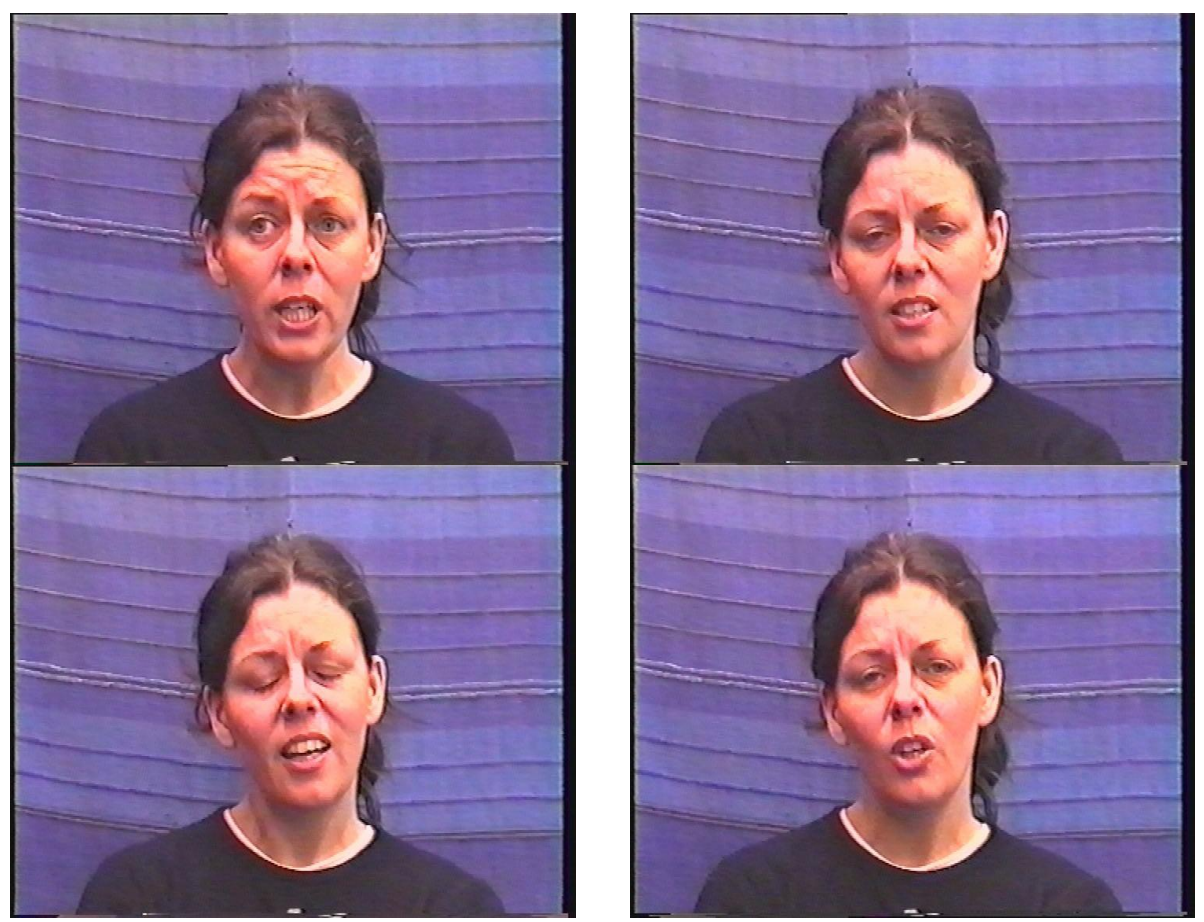

Fig. 1. Frames from the SAL dataset

\section{Extraction of Facial Features}

An overview of the proposed methodology is illustrated in Fig. 2. The face is first located, so that approximate facial feature locations can be estimated from the head position and rotation. Face roll rotation is estimated and corrected and the head is segmented focusing on the following facial areas: left eye/eyebrow, right eye/eyebrow, nose and mouth. Each of those areas, called feature-candidate areas, contains the features whose boundaries need to be extracted for our purposes. Inside the corresponding feature-candidate areas precise feature extraction is performed for each facial feature, i.e. eyes, eyebrows, mouth and nose, using a multi-cue approach, generating a small number of intermediate feature masks. Feature masks generated for each facial feature are fused together to produce the final mask for that feature. The mask fusion process uses anthropometric criteria [18] to perform validation and weight assignment on each intermediate mask; each feature's weighted masks are then fused to produce a final mask along with confidence level estimation.

Since this procedure essentially locates and tracks points in the facial area, we chose to work with MPEG-4 FAPs and not Action Units (AUs), since the former are explicitly defined to measure the deformation of these feature points. In addition to this, discrete points are easier to track in cases of extreme rotations and their position 


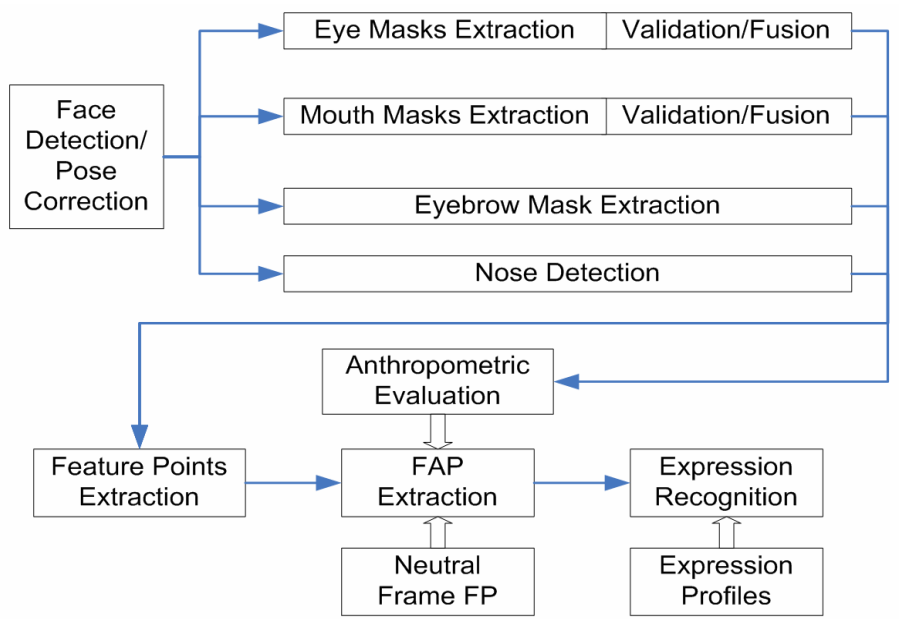

Fig. 2. High-level overview of the facial feature extraction process

can be estimated based on anthropometry in cases of occlusion, whereas this is not usually the case with whole facial features. Another feature of FAPs which proved useful is their value (or magnitude) which is crucial in order to differentiate cases of varying activation of the same emotion (e.g. joy and exhilaration) [5] and exploit fuzziness in rule-based systems [50]. Measurement of Facial Animation Parameters (FAPs) requires the availability of a frame where the subject's expression is found to be neutral. This frame will be called the neutral frame and is manually selected from video sequences to be analyzed or interactively provided to the system when initially brought into a specific user's ownership. The final feature masks are used to extract 19 Feature Points (FPs) [5]; Feature Points obtained from each frame are compared to FPs obtained from the neutral frame to estimate facial deformations and produce the Facial Animation Parameters (FAPs). Confidence levels on FAP estimation are derived from the equivalent feature point confidence levels. The FAPs are used along with their confidence levels to provide the facial expression estimation.

\subsection{Face Detection and Pose Estimation}

In the proposed approach facial features including eyebrows, eyes, mouth and nose are first detected and localized. Thus, a first processing step of face detection and pose estimation is carried out as described below, to be followed by the actual facial feature extraction process described in the following section. At this stage, it is assumed that an image of the user at neutral expression is available, either a-priori, or captured before interaction with the proposed system starts.

The goal of face detection is to determine whether or not there are faces in the image, and if yes, return the image location and extent of each face [30]. Face detection can be performed with a variety of methods. In this paper we used nonparametric discriminant analysis with a Support Vector Machine (SVM) which classifies face and non-face areas reducing the training problem dimension to a fraction of the original with negligible loss of classification performance [44],[10]. 


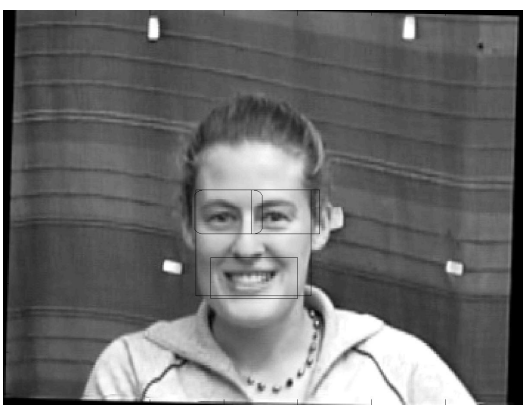

(a)

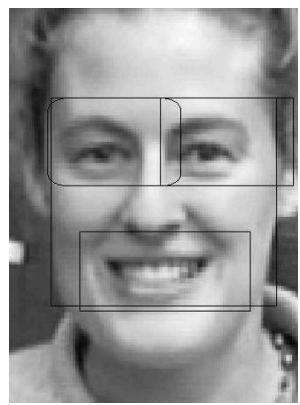

(b)

Fig. 3. Feature-Candidate areas: (a) full frame (352x288). (b) zoomed (90x125).

The face detection step provides a rectangle head boundary which includes all facial features as shown in Fig. 3. The latter can be then segmented roughly using static anthropometric rules into three overlapping rectangle regions of interest which include both facial features and facial background; these three feature-candidate areas include the left eye/eyebrow, the right eye/eyebrow and the mouth. In the following we utilize these areas to initialize the feature extraction process. Scaling does not affect feature-candidate area detection, since the latter is proportional to the head boundary extent, extracted by the face detector.

The accuracy of feature extraction depends on head pose. In this paper we are mainly concerned with roll rotation, since it is the most frequent rotation encountered in real life video sequences. Small head yaw and pitch rotations which do not lead to feature occlusion do not have a significant impact on facial expression recognition. The face detection techniques described in the former section is able to cope with head roll rotations up to $30^{\circ}$. This is a quite satisfactory range in which the featurecandidate areas are large enough so that the eyes reside in the eye-candidate search areas defined by the initial segmentation of a rotated face.

To estimate the head pose we first locate the left and right eyes in the detected corresponding eye candidate areas. After locating the eyes, we can estimate head roll rotation by calculating the angle between the horizontal plane and the line defined by the eye centers. To increase speed and reduce memory requirements, the eyes are not detected on every frame using the neural network. Instead, after the eyes are located in the first frame, two square grayscale eye templates are created, containing each of the eyes and a small area around them. The size of the templates is half the eye-center distance (bipupil breadth, $D_{b p}$ ). For the following frames, the eyes are located inside the two eye-candidate areas, using template matching which is performed by finding the location where the sum of absolute differences (SAD) is minimized.

After head pose is computed, the head is rotated to an upright position and new feature-candidate segmentation is performed on the head using the same rules so as to ensure facial features reside inside their respective candidate regions. These regions containing the facial features are used as input for the facial feature extraction stage, described in the following section. 


\subsection{Automatic Facial Feature Detection and Boundary Extraction}

To be able to compute MPEG-4 FAPs, precise feature boundaries for the eyes, eyebrows and mouth have to be extracted. Eye boundary detection is usually performed by detecting the special color characteristics of the eye area [47], by using luminance projections, reverse skin probabilities or eye model fitting. Mouth boundary detection in the case of a closed mouth is a relatively easily accomplished task. In case of an open mouth, several methods have been proposed which make use of intensity or color information. Color estimation is very sensitive to environmental conditions, such as lighting or capturing camera's characteristics and precision. Model fitting usually depends on ellipse or circle fitting, using Hough-like voting or corner detection [23]. Those techniques while providing accurate results in high resolution images, are unable to perform well with low video resolution which lack high frequency properties; such properties which are essential for efficient corner detection and feature border trackability [8], are usually lost due to analogue video media transcoding or low quality digital video compression.

In this work, nose detection and eyebrow mask extraction are performed in a single stage, while for eyes and mouth which are more difficult to handle, multiple (four in our case) masks are created taking advantage of our knowledge about different properties of the feature area; the latter are then combined to provide the final estimates as shown in Fig. 4. More technical details can be found at [50].

\subsubsection{Eye Boundary Detection}

Luminance and color information fusion mask tries to refine eye boundaries extracted by the neural network described earlier building on the fact that eyelids usually appear darker than skin due to eyelashes and are almost always adjacent to the iris. The initial mask provided by the neural network is thresholded and the distance transformation of the resulting mask gives as the first eye mask.

This second approach is based on eyelid edge detection. Eyelids reside above and below the eye centre, which has already been estimated by the neural network. Taking advantage of their mainly horizontal orientation, eyelids are easily located through edge detection. By combining the canny edge detector and the vertical gradient we are locating the eyelids and the space between them is considered the eye mask.

A third mask is created for each of the eyes to strengthen the final mask fusion stage. This mask is created using a region growing technique; the latter usually gives very good segmentation results corresponding well to the observed edges. Construction of this mask relies on the fact that facial texture is more complex and darker inside the eye area and especially in the eyelid-sclera-iris borders, than in the areas around them. Instead of using an edge density criterion, we developed a simple but effective iterative method to estimate both the eye centre and eye mask based on the standard deviation of the luminance channel.

Finally, a second luminance-based mask is constructed for eye/eyelid border extraction. In this mask, we compute the normal luminance probability resembling to the mean luminance value of eye area defined by the NN mask. From the resulting 


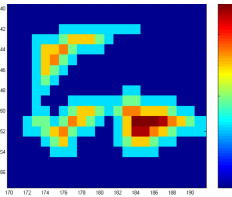

(a)

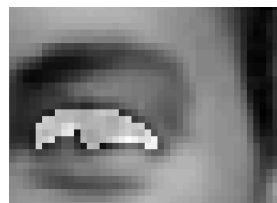

(b)

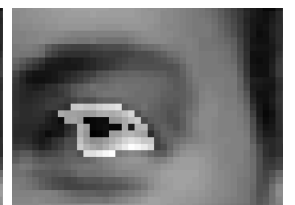

(c)

Fig. 4. Eye masks

probability mask, the areas with a given confidence interval are selected and small gaps are closed with morphological filtering. The result is usually a blob depicting the boundaries of the eye. In some cases, the luminance values around the eye are very low due to shadows from the eyebrows and the upper part of the nose. To improve the outcome in such cases, the detected blob is cut vertically at its thinnest points from both sides of the eye centre; the resulting mask's convex hull is used as the Luminance mask (Figure 4).

\subsubsection{Eyebrow Boundary Detection}

Eyebrows are extracted based on the fact that they have a simple directional shape and that they are located on the forehead, which due to its protrusion, has a mostly uniform illumination. Each of the left and right eye and eyebrow-candidate images shown in Figure 3 is used for brow mask construction.

The first step in eyebrow detection is the construction of an edge map of the grayscale eye/eyebrow-candidate image. This map is constructed by subtracting the dilation and erosion of the grayscale image using a line structuring element of size $n$ and then thresholding the result. The selected edge detection mechanism is appropriate for eyebrows because it can be directional; it preserves the feature's original size and can be combined with a threshold to remove smaller skin anomalies such as wrinkles. The above procedure can be considered as a non-linear high-pass filter.

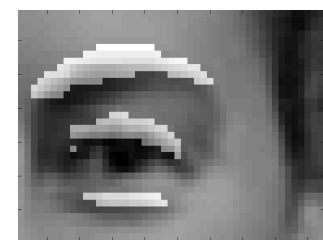

(a)

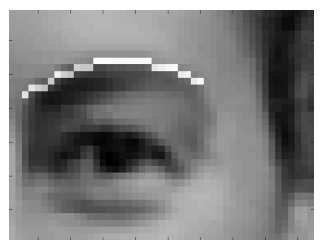

(b)

Fig. 5. (a) eyebrow-candidates. (b) selected eyebrow mask.

Each connected component on the edge map is labeled and then tested against a set of filtering criteria. These criteria were formed through statistical analysis of the eyebrow lengths and positions on 20 persons of the ERMIS SAL database [10]. Firstly, the major axis is found for each component through principal component analysis (PCA). All components whose major axis has an angle of more than 30 degrees with the horizontal plane are removed from the set. From the remaining components, those whose axis length is smaller than a given threshold are removed. 
Finally components with a lateral distance from the eye centre greater than a threshold calculated by anthropometric criteria are removed and the top-most remaining is selected resulting in the eyebrow mask (see Fig. 5).

\subsubsection{Nose Localization}

The nose is not used for expression estimation by itself, but is a fixed point that facilitates distance measurements for FAP estimation, thus, its boundaries do not have to be precisely located. Nose localization is a feature frequently used for face tracking and usually based on nostril localization; nostrils are easily detected based on their low intensity. Since inter-ocular distance in our images in the order of 50 pixels, nostril motion is limited, making them almost fixed and a good choice for a reference point.

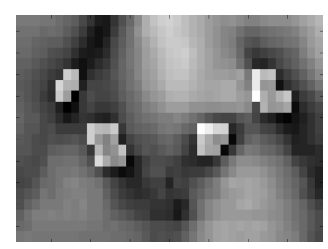

(a)

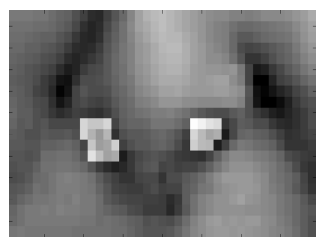

(b)

Fig. 6. (a) nostril candidates. (b) selected nostrils.

The facial area above the mouth-candidate components area is used for nose location. The respective luminance image is thresholded and connected objects of the derived binary map are labeled. In bad lighting conditions, long shadows may exist along either side of the nose. For this reason, anthropometric data about the distance of left and right eyes (bipupil breadth, etc.) is used to reduce the number of candidate objects. This has proven to be an effective way to remove most outliers without causing false negative results while generating the nostril mask shown in Figure 6a.

Horizontal nose coordinate is predicted from the coordinates of the two eyes. Each of the connected component horizontal distances from the predicted nose centre is compared to the average inter-nostril distance and components with the largest ones are considered as outliers. Those who qualify enter two separate lists, one including left-nostril candidates and one with right-nostril candidates based on their proximity to the left or right eye. Those lists are sorted according to their luminance and the two objects with the lowest values are retained from each list. The largest object is finally kept from each list and labeled as the left and right nostril respectively, as shown in Figure $6 \mathrm{~b}$. The nose centre is defined as the midpoint of the nostrils.

\subsubsection{Mouth Detection}

At first, mouth boundary extraction is performed on the mouth-candidate facial area depicted in Figure 3. An MLP neural network is trained to identify the mouth region using the neutral image. Since the mouth is closed in the neutral image, a long lowluminance region exists between the lips. The initial mouth-candidate luminance image is simplified to reduce the presence of noise, remove redundant information and produce a smooth image that consists mostly of flat and large regions of interest. 
Alternating Sequential Filtering by Reconstruction (ASFR) is thus performed on the initial mouth mask to produce a filtered image. ASFR ensures preservation of object boundaries through the use of connected operators [25]. The major axis of each connected component is computed through PCA analysis, and the one with the longest axis is selected. The latter is subsequently dilated vertically and this procedure results in a mask which includes the lips. The neural network trained on the neutralexpression frame, is then used on other frames to produce an estimate of the mouth area: neural network output on the mouth-candidate image is thresholded and those areas with high confidence are kept, to form a binary map containing several small sub-areas. The convex hull of these areas is calculated to generate the first final mask.

The second approach which produces a generic edge connection mask, the mouth luminance channel is again filtered using ASFR for image simplification. The horizontal morphological gradient of the original mouth mask is calculated similarly to the eyebrow binary edge map detection resulting in an intermediate mask. Since the nose has already been detected, its vertical position is known. The connected elements of the intermediate mask are labeled and those too close to the nose are removed. From the rest of the map, very small objects are removed by thresholding. Morphological closing is then performed and the longest of the remaining objects in horizontal sense is selected as the second mouth mask.

The problem of most intensity-based methods, that try to estimate mouth opening, is existence of upper teeth, i.e., those appearing between the upper and lower lip altering saturation and intensity uniformity. A new method is proposed next to cope with this problem. First, the mouth-candidate luminance channel is thresholded using a low threshold providing an estimate of the mouth interior area, or the area between the lips in case of a closed mouth. The threshold used is estimated adaptively.

In the resulting binary map, all connected objects adjacent to the border are removed. We now examine two cases separately: either we have no apparent teeth and the mouth area is denoted by a cohesive dark area (case 1) or teeth are apparent and thus two dark areas appear at both sides of the teeth (case 2). It should be noted that those areas appear even in large extensive smiles. The largest connected object is then selected and its centroid is found. If the horizontal position of its centroid is near the horizontal nose position case 1 is selected, otherwise case 2 is assumed to occur and two dark areas appear at both sides of the teeth. The two cases are quite distinguishable through this process. In case 2, the second largest connected object is also selected. A new binary map is created containing either one object in case 1 or both objects in case 2 ; the convex hull of this map is then calculated.

The detected lip corners provide a robust estimation of mouth horizontal extent but are not adequate to detect mouth opening. Therefore, the latter binary mask is expanded to include the lower lips. An edge map is created as follows: the mouth image gradient is calculated in the horizontal direction, and is thresholded by the median of its positive values. This mask contains objects close to the lower middle part of the mouth, which are sometimes missed because of the lower teeth. The two masks have to be combined to a final mask. An effective way of achieving this is to keep from both masks objects which are close to each other. 


\subsection{Final Masks Generation and Confidence Estimation}

Each facial feature's masks must be fused together to produce a final mask for that feature. The most common problems, especially encountered in low quality input images, include connection with other feature boundaries or mask dislocation due to noise. In some cases some masks may have completely missed their goal and provide a completely invalid result. Outliers such as illumination changes and compression artifacts cannot be predicted and so individual masks have to be re-evaluated and combined on each new frame.

The proposed algorithms presented in the previous sections produce a mask for each eyebrow, nose coordinates, four intermediate mask estimates for each eye and three intermediate mouth mask estimates. The four masks for each eye and three mouth masks must be fused to produce a final mask for each feature. Since validation can only be done on the end result of each intermediate mask, we unfortunately cannot give different parts of each intermediate mask different confidence values, so each pixel of those masks will share the same value (see Fig. 7). We propose validation through testing against a set of anthropometric conformity criteria. Since, however some of these criteria relate either to aesthetics or to transient feature properties, we cannot apply strict anthropometric judgment.
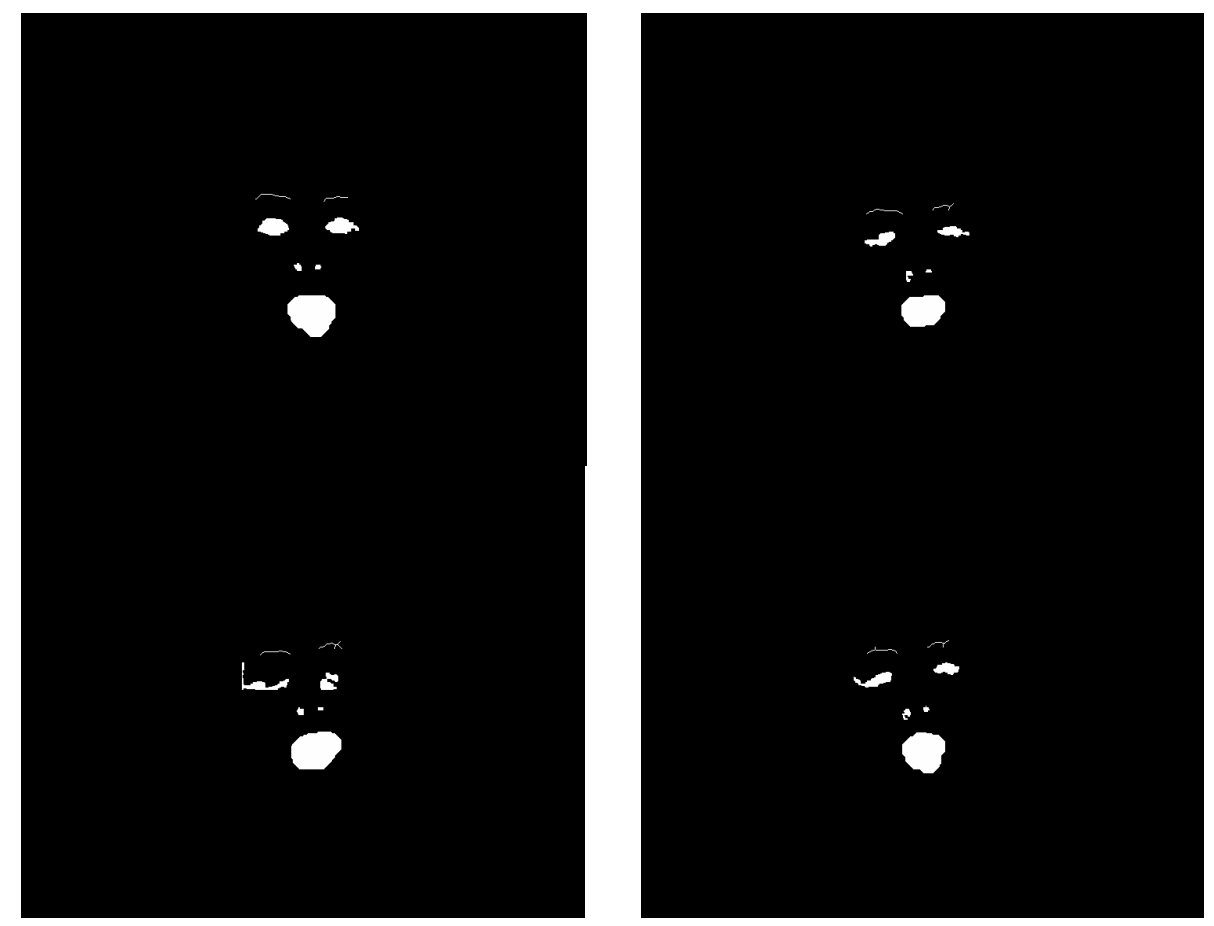

Fig. 7. Final masks for the frames shown in Fig. 1 
For each mask of every feature, we employ a set of validation measurements, which are then combined to a final validation tag for that mask. Each measurement produces a validation estimate value depending on how close it is to the usually expected feature shape and position, in the neutral expression. Expected values for these measurements are defined from anthropometry data [18] and from images extracted from video sequences of 20 persons in our database [10]. Thus, a validation tag between $[0,1]$ is attached to each mask, with higher values denoting proximity to the most expected measurement values. We want masks with very low validation tags to be discarded from the fusion process and thus those are also prevented from contribution on final validation tags.

\subsection{From FP to FAP Estimation}

A 25-dimensional distance vector is created containing vertical and horizontal distances between 19 extracted FPs, as shown in Fig. 8. Distances are normalized using scale-invariant MPEG-4 units, i.e. ENS, MNS, MW, IRISD and ES 36. Unit bases are measured directly from FP distances on the neutral image; for example ES is calculated as $\left|\mathrm{FP}_{9}, \mathrm{FP}_{13}\right|$.

The distance vector is created once for the neutral-expression image and for each of the subsequent frames FAPs are calculated by comparing them with the neutral frame. The value of each FAP is calculated from a set of geometric rules based on variations of distances from immovable points on the face. For example, the inner eyebrow FAPs are calculated by projecting vertically the distance of the inner eye corners, points 8 and 12 in Figure 7, to points 3 and 6 and comparing it to their distance in the neutral frame. A more detailed discussion on this procedure is found at [5].

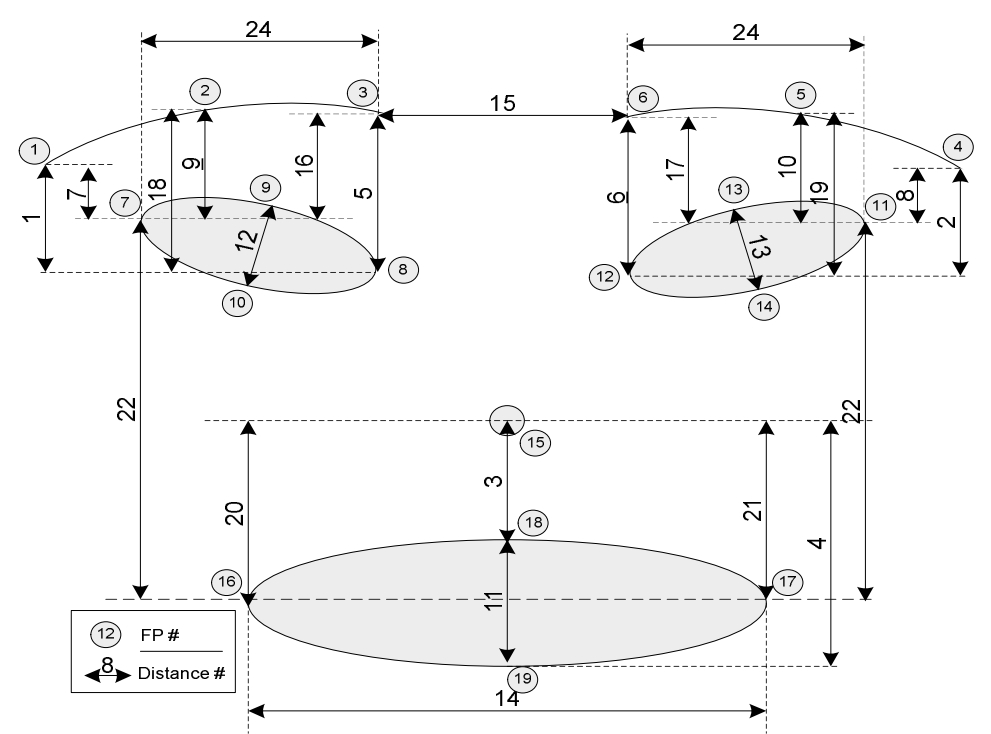

Fig. 8. Feature Point Distances 


\section{Hand Gesture Analysis}

In order to extract emotion-related features through hand movement, we implemented a lightweight hand-tracking subsystem [22, 51]. The purpose of this subsystem was not gesture recognition per se, but the extraction of quantitative parameters related to expressivity $[12,7]$; as a result, emphasis is on detailed tracking, e.g. in the case of occlusion, but on quick calculation of the position of the hands. The low-level part of the process involves the creation of moving skin masks, namely skin color areas which are tracked between subsequent frames. By tracking the centroid of those skin masks we produce an estimate of the user's movements, exploiting also the fact that in the

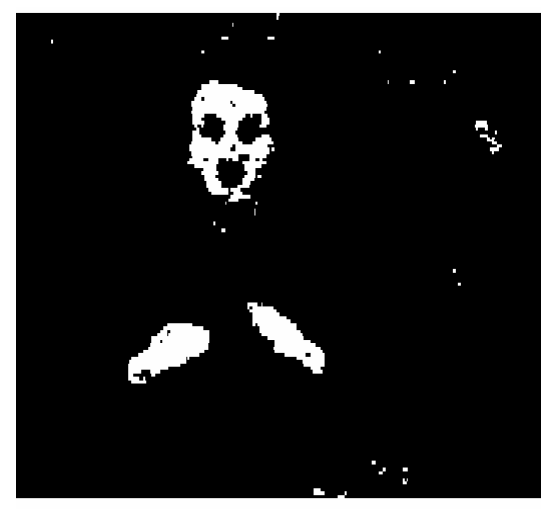

Fig. 9. Initial color mask created with skin detection

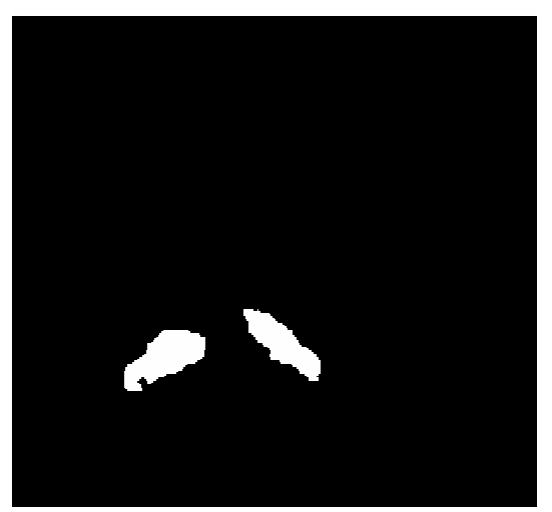

Fig. 11. Detected moving hand segments after morphological reconstruction

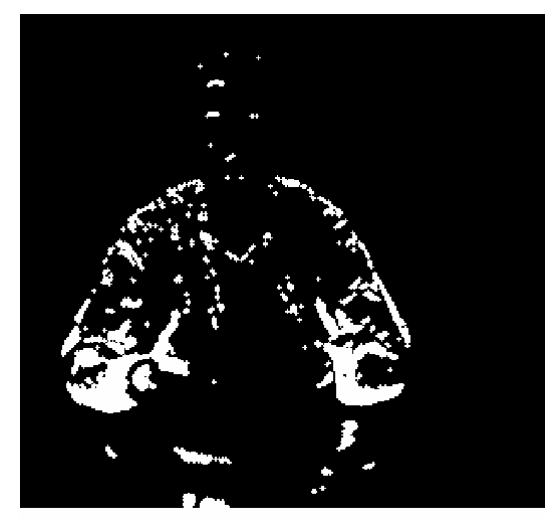

Fig. 10. Initial motion mask after pixel difference thresholded to $10 \%$ of maximum

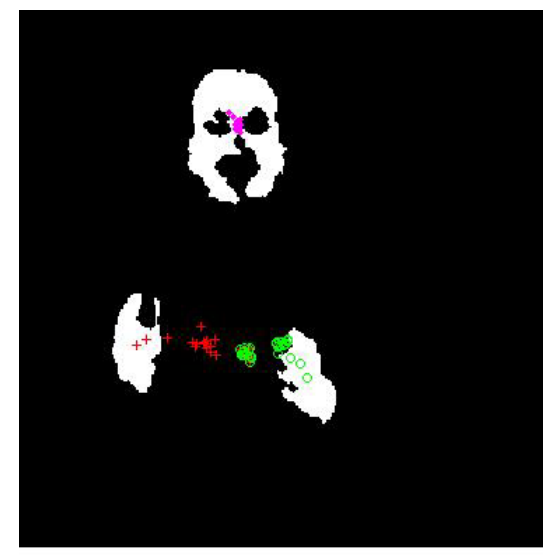

Fig. 12. Tracking of both hand objects in the "clapping" sequence 
context is MMI applications, one expects to locate the head in the middle area of upper half of the frame and the hand segments near the respective lower corners.

For each given frame, a skin color probability matrix is computed by calculating the joint thresholded probability of the $\mathrm{Cr} / \mathrm{Cb}$ image values (Fig. 9). Candidate areas for the 'possible-motion mask' are found by thresholding the difference pixels between the current frame and the next, resulting to Fig. 10; in essence, this mask is used to constrain processing only to moving, hence expressive, parts of the image. In the following, morphological filtering is employed on both masks in order to eliminate artifacts created by noise and background objects with color similar to the skin. The final moving skin mask is then created by fusing the processed skin and motion masks through the morphological reconstruction of the color mask using the motion mask as marker. The result of this process, after excluding the head object is shown in Fig. 11. The moving skin mask consists of many large connected areas. For the next frame a new moving skin mask is created, and a one-to-one object correspondence is performed. Object correspondence between two frames is performed on the color mask and is based on object centroid distance for objects of similar (at least 50\%) area. In Fig. 12, red markers represent the position of the centroid of the detected right hand of the user, while green markers correspond to the left hand. In the case of hand object merging and splitting, e.g. in the case of clapping, we establish a new matching of the left-most candidate object to the user's right hand and the right-most object to the left hand.

The results of this first step are utilized to calculate the gesture expressivity parameters [7]. Table 2 shows the effect that each expressivity parameter has on the production of facial expressions and hand gestures. The Spatial Extent (SPC) parameter modulates the amplitude of the movement of arms, wrists (involved in the animation of a gesture), head and eyebrows (involved in the animation of a facial expression); it influences how wide or narrow their displacement will be during the final animation. For example let us consider the eyebrows rising in the expression of surprise: if the value of the Spatial Extent parameter is very high the final position of the eyebrows will be very high in the forehead (i.e. the eyebrows move under a strong of muscular contraction). The Temporal Extent (TMP) parameter shortens or lengthens the motion of the preparation and retraction phases of the gesture as well as the onset and offset duration for facial expression. On of the effect on the face is to speed up or slow down the rising/lowering of the eyebrows. The agent animation is generated by defining some key frames and computing the interpolation curves passing through these frames. The Fluidity (FLT) and Power (PWR) parameters act on the interpolation curves. Fluidity increases/reduces the continuity of the curves allowing the system to generate more/less smooth animations. Let us consider its effect on the head: if the value of the Fluidity parameter is very low the resulting curve of the head movement will appear as generated through linear interpolation. Thus, during its final animation the head will have a jerky movement. Power introduces a gesture/expression overshooting, that is a little lapse of time in which the body part involved by the gesture reaches a point in space further than the final one. For example the frown displayed in the expression of anger will be stronger for a short period of time, and then the eyebrows will reach the final position. The last parameter, Repetition (REP), exerts an influence on gestures and head movements. It increases the number of stroke of gestures to obtain repetition of the gestures 
Table 2. Expressivity parameters and effect on low-level features

\begin{tabular}{|c|c|c|c|}
\hline & HEAD & $\begin{array}{l}\text { FACIAL } \\
\text { EXPRESSION }\end{array}$ & GESTURE \\
\hline SPC & $\begin{array}{l}\text { wider/narrower } \\
\text { movement }\end{array}$ & $\begin{array}{l}\text { increased/decreased } \\
\text { muscular contraction }\end{array}$ & $\begin{array}{l}\text { wider/narrower } \\
\text { movement }\end{array}$ \\
\hline TMP & $\begin{array}{l}\text { shorter/longer } \\
\text { movement speed }\end{array}$ & $\begin{array}{l}\text { shorter/longer } \\
\text { onset and offset }\end{array}$ & $\begin{array}{l}\text { shorter/longer speed } \\
\text { of preparation and } \\
\text { retraction phases }\end{array}$ \\
\hline FLT & $\begin{array}{l}\text { increases/reduces } \\
\text { continuity of } \\
\text { head movement }\end{array}$ & $\begin{array}{l}\text { increases/reduces } \\
\text { continuity of } \\
\text { muscular contraction }\end{array}$ & $\begin{array}{l}\text { increases/reduces } \\
\text { continuity between } \\
\text { consecutive gestures }\end{array}$ \\
\hline PWR & $\begin{array}{l}\text { higher/shorter } \\
\text { head } \\
\text { overshooting }\end{array}$ & $\begin{array}{l}\text { higher/shorter } \\
\text { muscular contraction } \\
\text { overshooting }\end{array}$ & $\begin{array}{l}\text { more/less } \\
\text { stroke } \\
\text { acceleration }\end{array}$ \\
\hline REP & $\begin{array}{l}\text { more/less } \\
\text { number of } \\
\text { nods and shakes }\end{array}$ & $\begin{array}{l}\text { not } \\
\text { implemented } \\
\text { yet }\end{array}$ & $\begin{array}{l}\text { more/less } \\
\text { number of repetitions } \\
\text { of the same stroke }\end{array}$ \\
\hline
\end{tabular}

themselves in the final animation. Let us consider the gesture "wrists going up and down in front of the body with open hands and palms up", a high value of the Repetition parameter will increase the number of the up and down movements. On the other hand this parameter decreases the time period of head nods and head shakes to obtain more nods and shakes in the same lapse of time.

\section{Extraction of Acoustic Features}

The features used in this work are exclusively based on prosody and related to pitch and rhythm. All information related to emotion that one can extract from pitch is probably not only in these features, but the motivation of this approach is in the desire to develop and use a high level of speech prosody analysis, calculate as many features as possible and then reduce them to those uncorrelated with each other and relevant to expressivity ([42], [40]).

We analyzed each tune with a method employing prosodic representation based on perception called 'Prosogram' [39]. Prosogram is based on a stylization of the fundamental frequency data (contour) for vocalic (or syllabic) nuclei. It gives globally for each voiced nucleus a pitch and a length. According to a 'glissando treshold' in some cases we don't get a fixed pitch but one or more lines to define the evolution of pitch for this nucleus. This representation is in a way similar to the 'piano roll' representation used in music sequencers. This method, based on the Praat environment, offers the possibility of automatic segmentation based both on voiced part and energy maxima. From this model - representation stylization we extracted several types of features: pitch interval based features, nucleus length features and distances between nuclei.

In musical theory, ordered pitch interval is the distance in semitones between two pitches upwards or downwards. For instance, the interval from $\mathrm{C}$ to $\mathrm{G}$ upward is 7 , 
but the interval from $\mathrm{G}$ to $\mathrm{C}$ downwards is -7 . Using integer notation (and eventually modulo 12) ordered pitch interval, ip, may be defined, for any two pitches $x$ and $y$, as:

$$
\begin{aligned}
& i p\langle y, x\rangle=x-y \\
& i p\langle x, y\rangle=y-x
\end{aligned}
$$

In this study we considered pitch intervals between successive voiced nuclei. For any two pitches $x$ and $y$, where $x$ precedes $y$, we calculate the interval ip $\langle x, y\rangle=y-x$, then deduce the following features.

For each tune, feature (f1) is the minimum of all the successive intervals in the tune. In a similar way, we extract the maximum (f2), the range (absolute difference between minimum and maximum) (f3), of all the successive intervals in each tune. Using the same measure, we also deduce the number of positive intervals (f4) and the number of negative intervals (f5). Using the absolute value, a measure equivalent to the unordered pitch interval in music theory, we deduce a series of similar features: minimum (f6), maximum (f7), mean (f8) and range (f9) of the pitch interval. Another series of features is also deduced from the ratio between successive intervals, here again maximum (f10), minimum (f11), mean (f12) and range (f13) of these ratios give the related features. In addition to the aforementioned features, the usual pitch features have also been used such as fundamental frequency minimum (f14), maximum (f15), mean (f16) and range (f17). The global slope of the pitch curve (f18), using linear regression, has also been added.

As was previously said, each segment (voiced 'nucleus' if it is voiced) of this representation has a length, and this has also been used in each tune to extract features related to rhythm. These features are, as previously, maximum (f19), minimum (f20), mean (f21) and range (f22). Distances between segments have also been used as features and the four last features we used are maximum (f23), minimum (f24), mean (f25) and range (f26) of these distances.

\section{Fusion of Visual and Acoustic Features}

As primary material we consider the audiovisual content collected using the SAL approach. This material was labeled using FeelTrace [43] by four labelers. The activation-valence coordinates from the four labelers were initially clustered into quadrants [42] and were then statistically processed so that a majority decision could be obtained about the unique emotion describing the given moment. The corpus under investigation was segmented into 1000 tunes of varying length. For every tune, the input vector consisted of the FAPs and expressivity parameters produced by the processing of the frames of the tune plus one value per SBPF (Segment Based Prosodic Feature) per tune. The fusion was performed on a frame basis, meaning that the values of the SBPFs were repeated for every frame of the tune. This approach was preferred because it preserved the maximum of the available information since SBPFs are only meaningful for a certain time period and cannot be calculated per frame. 
The implementation of a RNN we used was based on an Elman network [20], [35]. The input vectors were formed as described earlier and the output classes were 4 ( 3 for the possible emotion quadrants, since the data for the positive/passive quadrant was negligible, and one for neutral affective state) resulting in a dataset consisting of around 13,000 records. The classification efficiency for the unimodal case was measured at $67 \%$ (visual) and $73 \%$ (prosody), but combining all modalities increased mean recognition rate to $82 \%$. As is illustrated in Fig. 13, the ability of the network to adapt to and overcome possible feature extraction errors in a single frame rises with the length of the tune in question, reaching more than $85 \%$ for tunes lasting more than a few seconds. Besides this, the network shows impressive ability to differentiate between half planes, especially in the case of activation (active vs. passive emotions).

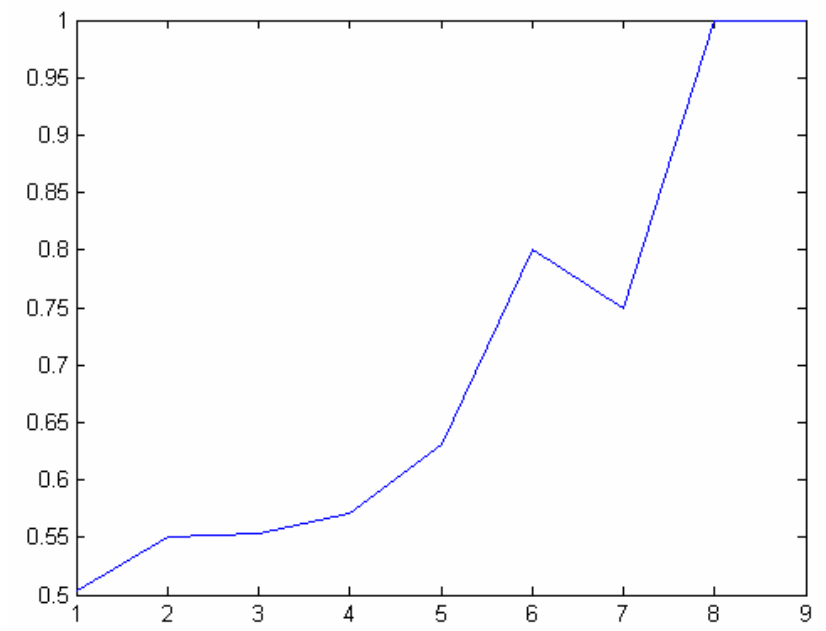

Fig. 13. Quadrant recognition rate over tune length (horizontal axis in intervals of $0.8 \mathrm{sec}$ )

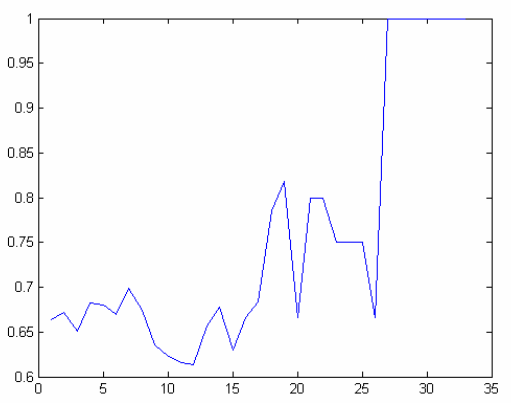

(a)

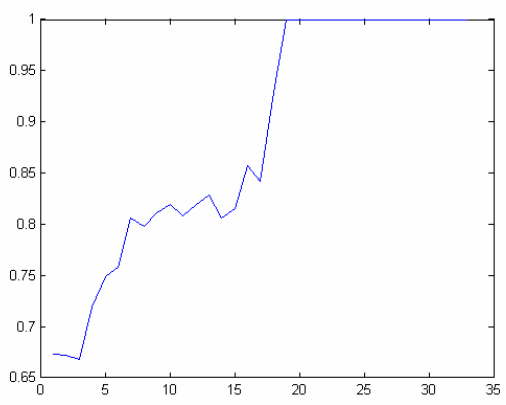

(b)

Fig. 14. (a) Positive-Negative recognition rate, (b) Active-Passive recognition rate, horizontal axis shows tune length in $0.2 \mathrm{sec}$ units 


\section{Conclusions - Future Work}

Naturalistic data goes beyond extreme emotions and concentrates on more natural emotional episodes that happen more frequently in everyday discourse. In this paper, we described a feature-based approach that tackles most of the intricacies of everyday audiovisual HCI and models the time-varying nature of these features in cases of expressivity. Most approaches focus on the detection of visual features in prerecorded, acted datasets and the utilization of machine learning algorithms to estimate the illustrated emotions. Even in cases of multimodality, features are fed into the machine learning algorithms without any real attempt to find structure and correlations between the features themselves and the estimated result. Neural networks are a nice solution to finding such relations, thus coming up with comprehensible connections between the input (features) and the output (emotion).

The fact that we use naturalistic and not acted data introduces a number of interesting issues, for example segmentation of the discourse in tunes. During the experiment, tunes containing a small number of frames (less than 5 frames, i.e. 0.2 seconds) were found to be error prone and classified close to chance level (not better than $37 \%$ ). This is attributed to the fact that emotion in the speech channel needs at least half a second to be expressed via wording, as well as to the internal structure of the Elman network which works better with a short-term memory of ten frames. From a labeling point of view, ratings from four labelers are available; in some cases, experts would disagree in more than $40 \%$ of the frames in a single tune. In order to integrate this fact, the decision system has to take into account the interlabeller disagreement, by comparing this to the level of disagreement with the automatic estimation. One way to achieve this, is the modification of the Williams Index [110], which is used to this effect for the visual channel in [50].

A future direction regarding the features themselves is to model the correlation between phonemes and FAPs. In general, feature points from the mouth area do not contribute much when the subject is speaking; however, consistent phoneme detection could help differentiate expression-related deformation (e.g. a smile) to speechrelated. Regarding the speech channel, the multitude of the currently detected features is hampering the training algorithms. To overcome this, we need to evaluate the importance/prominence of features so as to conclude on the influence they have on emotional transition.

Acknowledgments. This work has been funded by the FP6 Network of Excellence Humaine: Human-Machine Interaction Network on Emotion, URL:

http://www.emotion- research.net

\section{References}

1. A. Jaimes, Human-Centered Multimedia: Culture, Deployment, and Access, IEEE Multimedia Magazine, Vol. 13, No.1, 2006.

2. A. Mehrabian, Communication without Words, Psychology Today, vol. 2, no. 4, pp. 53-56, 1968. 
3. A. Nogueiras, A. Moreno, A. Bonafonte and J.B. Mariño, Speech emotion recognition using hidden markov models. Proceedings of Eurospeech, Aalborg, Denmark, 2001.

4. A. Pentland, Socially Aware Computation and Communication, Computer, vol. 38, no. 3, pp. 33-40, 2005.

5. A. Raouzaiou, N. Tsapatsoulis, K. Karpouzis and S. Kollias, Parameterized facial expression synthesis based on MPEG-4, EURASIP Journal on Applied Signal Processing, Vol. 2002, No 10, 2002, pp. 1021-1038.

6. A.J. Fridlund, Human Facial Expression: An Evolutionary Perspective, Academic Press, 1994.

7. B. Hartmann, M. Mancini, C. Pelachaud, Formational parameters and adaptive prototype instantiation for MPEG-4 compliant gesture synthesis, In Computer Animation'02, Geneva, Switzerland. IEEE Computer Society Press, 2002.

8. C. Tomasi and T. Kanade, Detection and Tracking of Point Features, Carnegie Mellon University Technical Report CMU-CS-91-132, April 1991.

9. F. Freitag, E. Monte, Acoustic-phonetic decoding based on Elman predictive neural networks, Proceedings of ICSLP 96, Fourth International Conference on, Page(s): 522525, vol.1.

10. FP5 IST ERMIS, Emotionally Rich Man-machine Intelligent System IST-2000-29319, http://www.image.ntua.gr/ermis

11. FP6 IST HUMAINE, Human-Machine Interaction Network on Emotion, 2004-2007, http://www.emotion-research.net

12. G. Caridakis, A. Raouzaiou, K. Karpouzis, S. Kollias. Synthesizing Gesture Expressivity Based on Real Sequences. Workshop on multimodal corpora: from multimodal behaviour theories to usable models, LREC 2006 Conference, Genoa, Italy, 24-26 May.

13. G. W. Williams, Comparing the joint agreement of several raters with another rater", Biometrics, vol32, pp. 619-627, 1976.

14. H. G. Zimmermann, R. Grothmann, A. M. Schaefer, and Ch. Tietz. Identification and forecasting of large dynamical systems by dynamical consistent neural networks. In S. Haykin, T. Sejnowski J. Principe, and J. McWhirter, editors, New Directions in Statistical Signal Processing: From Systems to Brain. MIT Press, 2006.

15. I. Cohen, A. Garg, and T. S. Huang, Emotion Recognition using Multilevel-HMM, NIPS Workshop on Affective Computing, Colorado, Dec 2000.

16. I. Cohen, N. Sebe, F. Cozman, M. Cirelo, and T.S. Huang. Learning Bayesian network classifiers for facial expression recognition using both labeled and unlabeled data. In Proc. Conf. on Computer Vision and Pattern Recognition, volume 1, pages 595-601, 2003.

17. J. Lien, Automatic recognition of facial expressions using hidden markov models and estimation of expression intensity, Ph.D. dissertation, Carnegie Mellon University, Pittsburg, PA, 1998.

18. J. W. Young, Head and Face Anthropometry of Adult U.S. Civilians, FAA Civil Aeromedical Institute, 1963-1993 (final report 1993)

19. J. Weizenbaum, ELIZA - A Computer Program For the Study of Natural Language Communication Between Man and Machine, Communications of the ACM, Volume 9, Number 1, 1966, pp. 36-35.

20. J.L. Elman, Finding structure in time, Cognitive Science, vol. 14, 1990, pp. 179-211.

21. K. Karpouzis, A. Raouzaiou, A. Drosopoulos, S. Ioannou, T. Balomenos, N. Tsapatsoulis and S. Kollias, Facial expression and gesture analysis for emotionally-rich man-machine interaction, in N. Sarris, M. Strintzis, (eds.), 3D Modeling and Animation: Synthesis and Analysis Techniques, pp. 175-200, Idea Group Publ., 2004. 
22. K. Karpouzis, A. Raouzaiou, A. Drosopoulos, S. Ioannou, T. Balomenos, N. Tsapatsoulis and S. Kollias, Facial expression and gesture analysis for emotionally-rich man-machine interaction, N. Sarris, M. Strintzis, (eds.), 3D Modeling and Animation: Synthesis and Analysis Techniques, pp. 175-200, Idea Group Publ., 2004.

23. K.M. Lam, H. Yan, Locating and Extracting the Eye in Human Face Images, Pattern Recognition, Vol.29, No.5, 1996, pp. 771-779.

24. K.R. Scherer, Adding the affective dimension: A new look in speech analysis and synthesis, In Proc. International Conf. on Spoken Language Processing, pp. 1808-1811, 1996.

25. L. Vincent, Morphological Grayscale Reconstruction in Image Analysis: Applications and Efficient Algorithms, IEEE Trans. Image Processing, vol. 2, no. 2, 1993, pp. 176-201.

26. L.C. De Silva and P.C Ng, Bimodal emotion recognition, In Proc. Automatic Face and Gesture Recognition, pp. 332-335, 2000.

27. L.M. Wang, X.H. Shi, G.J. Chen, H.W. Ge, H.P. Lee, Y .C. Liang, Applications of PSO Algorithm and OIF Elman Neural Network to Assessment and Forecasting for Atmospheric Quality, ICANNGA 2005, 2005

28. L.S. Chen and T.S. Huang, Emotional expressions in audiovisual human computer interaction, In Proc. International Conference on Multimedia and Expo, pp. 423-426, 2000.

29. L.S. Chen, Joint processing of audio-visual information for the recognition of emotional expressions in human-computer interaction, $\mathrm{PhD}$ thesis, University of Illinois at UrbanaChampaign, Dept. of Electrical Engineering, 2000.

30. M. H. Yang, D. Kriegman, N. Ahuja, Detecting Faces in Images: A Survey, IEEE Trans. on Pattern Analysis and Machine Intelligence, Vol.24(1), 2002, pp. 34-58.

31. M. Pantic and L.J.M. Rothkrantz, Automatic analysis of facial expressions: The state of the art. IEEE Trans. on Pattern Analysis and Machine Intelligence, 22(12):1424-1445, 2000.

32. M. Pantic and L.J.M. Rothkrantz, Towards an Affect-sensitive Multimodal HumanComputer Interaction, Proceedings of the IEEE, vol. 91, no. 9, pp. 1370-1390, 2003.

33. M. Pantic, Face for Interface, in The Encyclopedia of Multimedia Technology and Networking, M. Pagani, Ed., Idea Group Reference, vol. 1, pp. 308-314, 2005.

34. M. Pantic, N. Sebe, J. Cohn, T. Huang, Affective Multimodal Human-Computer Interaction, Proceedings of the 13th annual ACM international conference on Multimedia, pp. $669-676,2005$.

35. Mathworks, Manual of Neural Network Toolbox for MATLAB

36. Murat Tekalp, Joern Ostermann, Face and 2-D mesh animation in MPEG-4, Signal Processing: Image Communication 15, Elsevier, pp. 387-421, 2000.

37. N. Sebe, I. Cohen, T.S. Huang, Handbook of Pattern Recognition and Computer Vision, World Scientific, January 2005

38. P. Ekman and W. Friesen, Pictures of Facial Affect, Palo Alto, CA: Consulting Psychologists Press, 1978.

39. P. Mertens, The Prosogram: Semi-Automatic Transcription of Prosody based on a Tonal Perception Model. in B. Bel \& I. Marlien (eds.), Proc. of Speech Prosody, Japan, 2004.

40. P. Oudeyer, The production and recognition of emotions in speech: features and algorithms. International Journal of Human Computer Interaction, 59(1-2):157-183, 2003.

41. R. Cowie and E. Douglas-Cowie, Automatic statistical analysis of the signal and prosodic signs of emotion in speech. In Proc. International Conf. on Spoken Language Processing, pp. 1989-1992, 1996. 
42. R. Cowie, E. Douglas-Cowie, N.Tsapatsoulis, G. Votsis, S. Kollias, W. Fellenz and J. G. Taylor, Emotion Recognition in Human-Computer Interaction, IEEE Signal Processign Magazine, pp 33- 80, January 2001.

43. R. Cowie, E. Douglas-Cowie, S. Savvidou, E. McMahon, M. Sawey and M. Schröder, FEELTRACE: An instrument for recording perceived emotion in real time, ISCA Workshop on Speech and Emotion, Northern Ireland, pp. 19-24, 2000.

44. R. Fransens, Jan De Prins, SVM-based Nonparametric Discriminant Analysis, An Application to Face Detection, Ninth IEEE International Conference on Computer Vision, Volume 2, October 13 - 16, 2003.

45. R. W. Picard, Affective Computing, MIT Press, 1997.

46. R. W. Picard, Towards computers that recognize and respond to user emotion, IBM Syst. Journal, 39 (3-4), 705-719, 2000.

47. R.L. Hsu, M. Abdel-Mottaleb, Anil K. Jain, Face Detection in Color Images, IEEE Transactions on Pattern Analysis and Machine Intelligence, Vol.24, No.5, May 2002

48. R.W. Picard, E. Vyzas, and J. Healey, Toward machine emotional intelligence: Analysis of affective physiological state, IEEE Trans. on Pattern Analysis and Machine Intelligence, 23(10):1175-1191, 2001.

49. S. Haykin. Neural Networks: A Comprehensive Foundation. Macmillan, New York, 1994.

50. S. Ioannou, A. Raouzaiou, V. Tzouvaras, T. Mailis, K. Karpouzis, S. Kollias, Emotion recognition through facial expression analysis based on a neurofuzzy network, Neural Networks, Elsevier, Vol. 18, Issue 4, May 2005, pp. 423-435

51. T. Balomenos, A. Raouzaiou, S. Ioannou, A. Drosopoulos, K. Karpouzis, S. Kollias, Emotion Analysis in Man-Machine Interaction Systems, Samy Bengio, Hervé Bourlard (Eds.), Machine Learning for Multimodal Interaction, Lecture Notes in Computer Science, Vol. 3361, 2004, pp. 318 - 328, Springer-Verlag.

52. U. Williams, K. N. Stevens, Emotions and Speech: some acoustical correlates, JASA 52, pp. 1238-1250, 1972.

53. Z. Zeng, J. Tu, M. Liu, T.S. Huang, Multi-stream Confidence Analysis for Audio-Visual Affect Recognition, ACII 2005, pp. 964-971.

54. Z. Zeng, Y. Hu, G. Roisman, Y. Fu, T. Huang, "Audio-visual, Emotion Recognition in Adult Attachment Interview, this volume. 